haben 53 Autoren mitgearbeitet. Sie haben 70 Einzelbeiträge verfaßt, die etwa 2800 Seiten einnehmen. Die Leistung der Band-Herausgeber, die sich in diesen Zahlen widerspiegelt, ist um so bewundernswerter als die vermutlich 1957 durchgeführte Planung des Bandes, die Auswahl der Autoren und die Koordination der Einzelbeiträge ungewöhnlich geglückt erscheinen, auch im Vergleich zu weniger umfangreichen Bänden des Handbuchs.

Gewiß hat es lange gedauert bis der Band 15 erscheinen konnte (der Bandherausgeber, Anton Lang, spricht von „höherer Gewalt“), sicherlich sind manche der Artikel schon vor Jahren abgeschlossen worden und selbstverständlich kann man im Hinblick auf die Verteilung der Schwerpunkte und im Hinblick auf den relativen Umfang der Einzelbeiträge verschiedener Auffassung sein; es wäre aber, so glaubt der Referent, im Fall dieses Bandes kleinlich, durch eine Kritik an Details die großartige Leistung von LANG, der selber mehrere Artikel geschrieben hat, und das Engagement der übrigen Autoren zu schmälern. Eine Einschränkung allgemeiner Art muß der Referent indessen vornehmen.

Der vorliegende Band 15 enthält im wesentlichen Daten und theoretische Vorstellungen der „klassischen Entwicklungsphysiologie“ (einschließlich vieler Aspekte der descriptiven Entwicklungsgeschichte). Das neue Kapitel der Entwicklungsphysiologie, das man vielleicht mit dem Titel eines kürzlich erschienenen Buches von J. Bonner überschreiben kann: The Molecular Biology of Development, tritt in dem Band nur insofern in Erscheinung als Anton Lang in seiner „Einführung" einen knappen Ausblick zu den molekularen Aspekten von Genetik und Entwicklungsphysiologie skizziert: „Genetics and development physiology are theme and countertheme. The objective of genetics is to understand how the hereditary information of organisms is written, that of developmental physiology, how it is read".

Der Doppelband 15 stellt in der Tat einen gewissen Abschluß der klassischen Entwicklungsphysiologie dar. Gleichzeitig aber ist dieses Werk ein Fundament, von dem aus das Problem der Entwicklung mit den Metho- den und Begriffen der Molekularbiologie in Angriff genommen werden kann. Die zeitliche Coincidenz im Erscheinen dieses Doppelbands und des schmalen Buchs von J. Bonner kann als ein glücklicher Zufall angesehen werden. Zu einem Zeitpunkt, da in der Entwicklungsphysiologie die Genphysiologie, die Biochemie und die Biophysik in den Vordergrund treten, wird uns das Wissensgut der klassischen Entwicklungsphysiologie in einer großartigen Zusammenstellung geboten. In den Bänden 14, 15 und 16 des Handbuchs ist in der Tat ein riesiger Schatz an Information für jene zusammengestellt, die sich anschicken, die neue Stufe hin zur Molekularbiologie der Entwicklung zu nehmen. Mögen sie von diesem Schatz Gebrauch machen. Ohne eine genaue Kenntnis der descriptiven Entwicklungsgeschichte und ohne die Daten der klassischen Entwicklungsphysiologie - erarbeitet auf dem Niveau der Zellen, Gewebe und Organe - wird man kaum in der Lage sein, die genphysiologischen und molekularen Aspekte der Entwicklung bei vielzelligen, komplexen Systemen erfolgreich zu studieren.

Der Band ist - wie üblich bei den Springer-Publikationen - glänzend ausgestattet. Das Namenverzeichnis und das doppelsprachige Sachverzeichnis (etwa 140 Seiten) sind hervorragende Hilfen bei der Orientierung. - Bedauerlich, so möchte man sagen, ist an dem Band lediglich der Preis. Der Einzelpreis von DM 748, - wird es den meisten Fachwissenschaftlern verwehren, sich den Band privat anzuschaffen. Es ist aber auch zu befürchten, daß manche Institutsbibliotheken, deren Etat eng begrenzt ist, auf die Anschaffung verzichten müssen. Unter diesem Aspekt fragt sich der Referent, ob als Kompromiß ein reduzierter Umfang (und damit ein geringerer Preis) des Bandes 15 nicht möglich gewesen wäre. Eine solche Beschränkung hätte in erster Linie die Überschneidungen der Artikel, vor allem auch die Überschneidungen mit dem schon seit mehreren Jahren erschienenen Band 16, betreffen können. - Natürlich haben derlei kritische Bemerkungen jetzt keinen Sinn mehr. Es bleibt zu hoffen, daß auch der Band 15 trotz seines abschreckenden Preises die verdiente Verbreitung findet.

H. Монг, Freiburg i. Br.

\title{
BERICHTIG UNG
}

Auf Seite 1305, Band $\mathbf{2 0}$ b, muß es auf der linken Spalte, 5. Zeile von oben, heißen:

(1. Gleichung) statt $\mathrm{F}_{3}-\mathrm{C}-\mathrm{C}-\mathrm{O}-\mathrm{C}-\mathrm{CF}_{3} / \mathrm{CF}_{3}-\mathrm{C}-\mathrm{O}-\mathrm{C}-\mathrm{CF}_{3}$.

In der Tabelle, 3. Spalte: statt $v^{\prime}>\mathrm{C} \equiv \mathrm{O}$ muß es heißen $v>\mathrm{C}=0$. 